\title{
Immunochemical analysis of poly(ADP-ribosyl)ation in HaCaT keratinocytes induced by the mono-alkylating agent 2-chloroethyl ethyl sulfide (CEES): Impact of experimental conditions
}

\author{
Malgorzata Debiak ${ }^{\mathrm{a}, 1}$, Kirsten Lex ${ }^{\mathrm{a}}$, Viviane Ponath ${ }^{\mathrm{a}}$, Waltraud Burckhardt-Boer ${ }^{\mathrm{a}}$, \\ Horst Thiermann $^{\mathrm{b}}$, Dirk Steinritz ${ }^{\mathrm{b}, \mathrm{c}}$, Annette Schmidt ${ }^{\mathrm{b}}$, Aswin Mangerich ${ }^{\mathrm{a}}$, \\ Alexander Bürkle ${ }^{a, *}$ \\ ${ }^{a}$ University of Konstanz, Molecular Toxicology Group, Department of Biology, 78457 Konstanz, Germany \\ b Bundeswehr Institute of Pharmacology and Toxicology, 80937 Munich, Germany \\ ${ }^{\mathrm{c}}$ Walther Straub Institute of Pharmacology and Toxicology, 80336 Munich, Germany
}

- Biological effects of CEES treated cells are significantly influenced by treatment protocol.

- Optimized protocol for the treatment of HaCaT cells with CEES.

- CEES induces a dose and time dependent PARylation response in HaCaT cells.

- CEES induced PAR formation is predominantly due to the activation of PARP1.

\section{Keywords:}

Poly(ADP-ribose)

Poly(ADP-ribose) polymerases

Sulfur mustard

CEES

HaCaT keratinocytes

Solvent

\begin{abstract}
A B S T R A C T
Sulfur mustard (SM) is a bifunctional alkylating agent with a long history of use as a chemical weapon. Although its last military use is dated for the eighties of the last century, a potential use in terroristic attacks against civilians remains a significant threat. Thus, improving medical therapy of mustard exposed individuals is still of particular interest. PARP inhibitors were recently brought into the focus as a potential countermeasure for mustard induced pathologies, supported by the availability of efficient compounds successfully tested in cancer therapy.

PARP activation after SM treatment was reported in several cell types and tissues under various conditions; however, a detailed characterization of this phenomenon is still missing. This study provides the basis for such studies by developing and optimizing experimental conditions to investigate poly(ADP ribosyl)ation (PARylation) in HaCaT keratinocytes upon treatment with the monofunctional alkylating agent 2 chloroethyl ethyl sulfide ("half mustard", CEES). By using an immunofluorescence based approach, we show that optimization of experimental conditions with regards to the type of solvent, dilution factors and treatment procedure is essential to obtain a homogenous PAR staining in HaCaT cell cultures. Furthermore, we demonstrate that different CEES treatment protocols significantly influence the cytotoxicity profiles of treated cells. Using an optimized treatment protocol, our data reveals that CEES induces a dose and time dependent dynamic PARylation response in HaCaT cells that could be completely blocked by treating cells with the clinically relevant pharmacological PARP inhibitor ABT888 (also known as veliparib). Finally, siRNA experiments show that CEES induced PAR formation is predominantly due to the activation of PARP1. In conclusion, this study provides a detailed analysis of the CEES induced PARylation response in HaCaT keratinocytes, which forms an experimental basis to study the molecular mechanism of PARP1 activation and its functional consequences after mustard treatment in general.
\end{abstract}

\footnotetext{
* Corresponding author at: Molecular Toxicology Group, Department of Biology, University of Konstanz, D-78457 Konstanz, Germany.

E-mail address: alexander.buerkle@uni-konstanz.de (A. Bürkle).

${ }^{1}$ Current address: Federal Environment Agency, 06844 Dessau-Rosslau, Germany.
} 


\section{Introduction}

Chemical weapons have been repeatedly used in military conflicts, since their first release during World War I a hundred years ago (Debiak et al., 2009; Mangerich and Esser, 2014). Although, due to considerable international efforts chemical warfare became almost completely banned by the international community, the risk of potential use of chemical weapons by terrorists remains a constant threat. As the production of sulfur mustard (SM) does not require specialized technology and large stockpiles might be available in the regions affected, its use against civilians is a realistic danger (Kehe et al., 2009; Kehe and Szinicz, 2005). The latest events during the Syrian civil war leading to the release of sarin, chlorine gas, and potentially also SM proved that such a threat is more real than ever.

The first synthesis of SM falls into the middle of 19th century. Along with phosgene and chlorine, SM entered the battlefields of World War I. While, the exposure is rarely lethal, SM causes severe skin and eye lesions and acute respiratory pathologies. Since a specific antidote is still missing, patient treatment is symptomatic and requires intensive and costly medical care and long term hospitalization (Kehe et al., 2009). The need for the development of effective therapy has drawn the attention to the mechanism of action of SM induced toxicity. Despite intensive research endeav ors throughout the last decades, the detailed mechanism of SM induced toxicity remains unclear. It is commonly accepted that the exposure causes rapid formation of SM protein and DNA adducts, resulting in massive cell death and inflammation of local tissue (Debiak et al., 2009; Kehe et al., 2009). Early on, an involvement of poly(ADP ribose) polymerase 1 (PARP1) was reported [reviewed in (Debiak et al., 2009)]. It was postulated that PARP1 becomes activated upon SM treatment in an apurinic endonuclease dependent manner (Papirmeister et al., 1985). Moreover, the treatment of cells with the first generation PARP inhibitor 3 aminobenzamide $(3 \mathrm{AB})$ preserved cellular $\mathrm{NAD}^{+}$pools. Interest ingly, PARP inhibition did not influence cell survival in general, but shifted the mode of cell death, from necrotic to apoptotic cell death (Kehe et al., 2008; Meier et al., 1987; Papirmeister et al., 1985). It has been hypothesized that this shift is responsible for reduced mustard induced pathologies under conditions of PARP inhibition in a rodent animal model (Cowan et al., 2003).

PARP1 is a ubiquitously expressed protein playing key roles in genomic maintenance, chromatin organization, transcription, and the regulation of cell death (Mangerich and Bürkle, 2012; Rouleau et al., 2010). PARP1 binds to DNA strand breaks and by using NAD ${ }^{+}$ as a substrate, catalyzes the formation of homopolymeric branched chains of poly(ADP ribose) (PAR) of different length that are covalently bound to "acceptor" proteins at glutamate, aspartate or lysine residues. Strong activators of PARP1 are ionizing radiation, alkylating agents inducing small base modifications, and reactive oxygen species; leading to the induction of direct or indirect DNA strand breaks generated in the process of base excision repair. SM is a bifunctional alkylating agent. The reaction with DNA leads to rapid formation of bulky monoadducts and DNA cross links (Debiak et al., 2011). The majority of DNA alkylation consists of adducts at the $N 7$ position of guanine and $N 3$ position of adenine, accounting for $61 \%$ and $16 \%$ of total alkylations, respectively (Ludlum et al., 1994). DNA di adducts, on the other hand, represent about $15 \%$ of SM induced DNA lesions and are formed by reaction of one SM molecule with $N 7$ positions of two guanines in close proximity (Ludlum and Papirmeister, 1986). SM and its derivative CEES are not expected to induce DNA strand breaks directly, thus potential PARP1 activation by such compounds may be mediated by unusual DNA structures exhibiting PARP1 activating potential or by DNA strand breaks that arise as DNA repair intermediates by enzymatic processing of the original DNA lesion.

The objective of this study was to investigate PARP1 activation in immortalized human keratinocytes [i.e., HaCaT cells (Boukamp et al., 1988)] after treatment with the SM analogue CEES. CEES is a monoalkylating agent that has been widely used as a SM surrogate substance (Bennett et al., 2014; Inturi et al., 2011; Kehe et al., 2013; Wang et al., 2012), because its use is not subject to specific restrictions. It exhibits similar chemical properties compared to SM, however, in contrast to SM, CEES is a monofunctional alkylating agent, and therefore lacks any crosslinking activity. Presumably due to lack of capability to produce DNA crosslinks, CEES is about 10 times less toxic than SM after oral application in rodents (Wang et al., 2012). The profile of cellular and tissue damage for CEES is comparable to that of SM (Jain et al., 2011; Tewari Singh et al., 2009). Based on previously described CEES treatment protocols, an optimized treatment protocol was developed to study PARP1 activation by immunofluorescence microscopy on a single cell level. Our results revealed that the experimental protocol of CEES treatment significantly affects the quality and quantity of PAR formation and cytotoxicity in HaCaT cells. Moreover, we define optimal concentrations and incubation times for pharmacological PARP inhibition and siRNA experiments demonstrate that PARP1 is responsible for the bulk of PAR formation upon CEES treatment.

This study provides the basis for a broader characterization of sulfur and nitrogen mustard induced PARylation response and cellular consequences thereof, which is described in an accompa nying article (Mangerich et al., 2016).

\section{Material and methods}

\subsection{Cell lines and culture conditions}

The human keratinocyte cell line HaCaT was kindly provided by Dr. Petra Boukamp, German Cancer Research Center, Heidelberg, Germany (Boukamp et al., 1988). HaCaT cells were cultured in Dulbecco's Modified Eagle Medium (Invitrogen) containing $4.5 \mathrm{~g} / \mathrm{l}$ glucose, $0.58 \mathrm{~g} / \mathrm{l} \mathrm{L}$ glutamine and $0.11 \mathrm{~g} / \mathrm{l}$ sodium pyruvate supple mented with $10 \%$ fetal calf serum (PAA Laboratories), penicillin $(100 \mathrm{U} / \mathrm{ml})$, streptomycin $(100 \mathrm{U} / \mathrm{ml})$ (Invitrogen) in a humidified atmosphere at $37^{\circ} \mathrm{C}$ and $5 \% \mathrm{CO}_{2}$. Cells were passaged twice a week in a 1:6 or $1: 10$ ratio. For detachment, cells were incubated with $0.25 \%$ trypsin EDTA (Invitrogen) for $5 \mathrm{~min}$ at $37^{\circ} \mathrm{C}$.

\subsection{CEES treatment}

2 Chloroethyl ethyl sulfide (CEES) (Sigma) was diluted in 100\% DMSO (protocol \#1) or $95 \%$ ethanol $/ 0.5 \% \mathrm{HCl}(\mathrm{v} / \mathrm{v})$ (protocols $\mu$ 12\#3). Serial dilutions of CEES were prepared at concentrations of $100 \times$ of the final concentrations (protocols \#1 and \#3). Equal distribution of CEES in PBS was ensured by short but intensive vortexing or inverting the tube. The solvent was kept constant at a final concentration of $1 \%(\mathrm{v} / \mathrm{v})$. The cells were washed carefully with PBS pre warmed to $37^{\circ} \mathrm{C}$ and immediately treated with CEES diluted in pre warmed PBS to the final concentration. The $\mathrm{pH}$ of the medium was monitored after CEES addition and proved to remain stable. The treatment occurred for $1060 \mathrm{~min}$ at $37^{\circ} \mathrm{C}$ and $5 \% \mathrm{CO}_{2}$. Thereafter, cells were washed with PBS and supplemented with fresh cell culture medium. In preliminary experiments CEES in a 


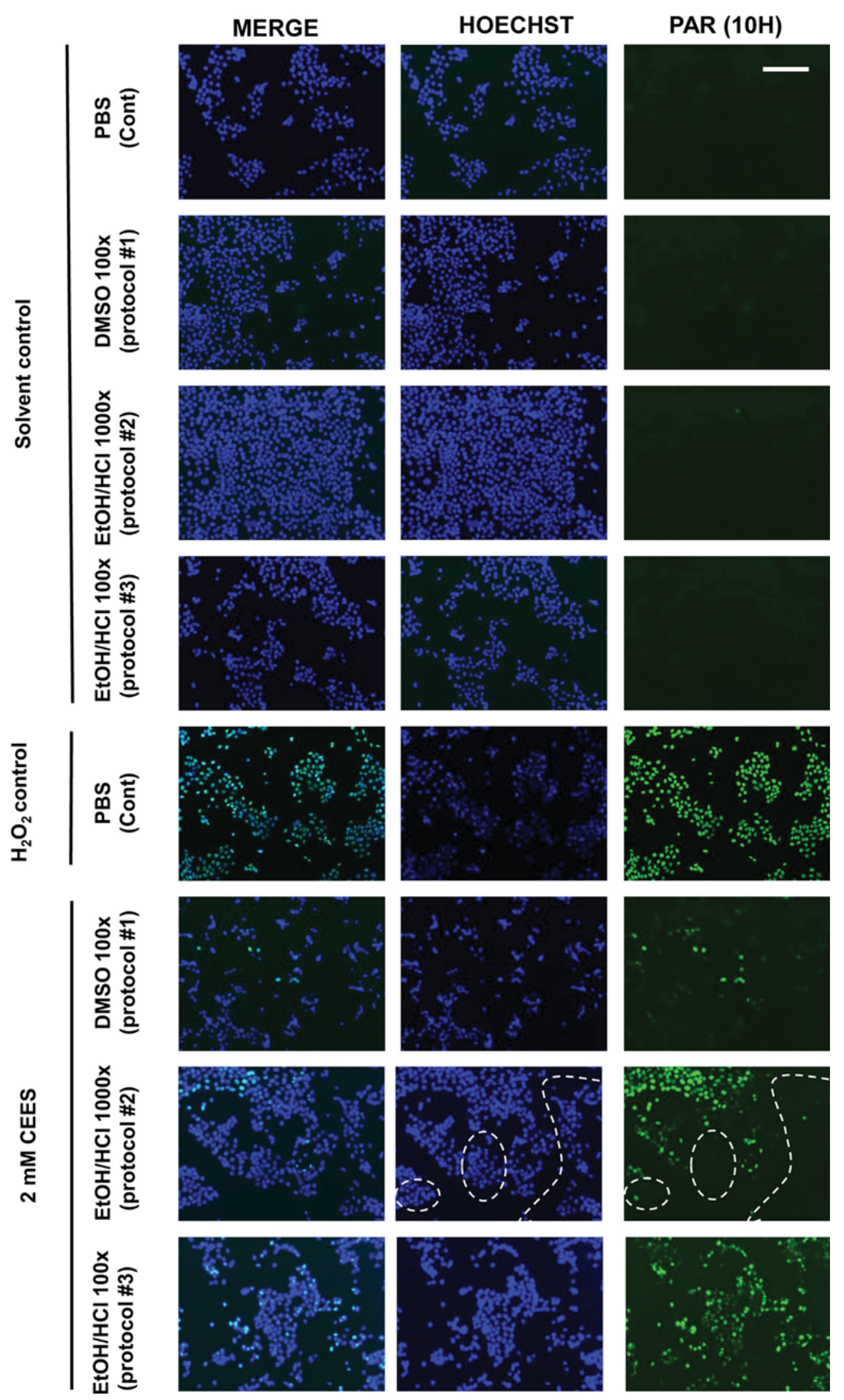

Fig. 1. CEES treatment protocol affects distribution of cellular PAR staining in HaCaT cell cultures.

Monolayers of HaCaT cells were treated for $10 \mathrm{~min}$ with $2 \mathrm{mM}$ CEES predisolved in different solvents and dilution factors as indicated (protocols \#1-3). CEES was either prediluted in $\mathrm{ETOH} / \mathrm{HCl}$ at a $1000 \times$ concentrated stock solution and added directly to the cell culture medium (protocol \#2) or in DMSO or EtOH/HCl in $100 \times$ concentrated stock solutions which were premixed in pre-warmed PBS, vortexed, and immediately applied to cells (protocols \#1 and \#3, respectively). PAR synthesis was analyzed by immuno-chemical staining using the PAR specific antibody $10 \mathrm{H}$ followed by epifluorescence analysis. $\mathrm{H}_{2} \mathrm{O}_{2}$ treatment for 5 min served as a positive control. Dashed lines in 
$1000 \times$ concentrated stock solution [95\% ethanol/0.5\% $\mathrm{HCl}(\mathrm{v} / \mathrm{v})]$ was added directly into the medium or PBS to the cell culture plates at a final concentration of the solvent of $0.1 \%(v / v)$ (protocol \#2). In order to distribute CEES equally in the culture medium, dishes were swiveled several times.

\subsection{Immunochemical analysis of cellular PAR formation}

Cells were grown on sterile glass cover slips and treated with CEES as described above. For detection of poly(ADP ribose), cells were fixed and permeabilized with ice cold methanol for $7 \mathrm{~min}$. The cover slips were washed $3 \times$ for 5 min with PBS and then blocked in $5 \%$ milk powder in PBS with $0.1 \%$ Tween 20 for 30 min at $30^{\circ} \mathrm{C}$. Specimens were incubated with the primary mAB $10 \mathrm{H}$ (Kawamitsu et al., 1984) over night at $4{ }^{\circ} \mathrm{C}$ in a humidified chamber, washed with PBS and incubated with secondary antibody (anti mouse Alexa 488) for $1 \mathrm{~h}$ at $37^{\circ} \mathrm{C}$ in the dark in a humidified chamber. Nuclei were visualized by Höchst 33342 (Molecular Probes, 1:20000 in PBS) staining for $5 \mathrm{~min}$. Specimens were washed with PBS, and slides were mounted in Aqua Polymount (Polysciences). Samples were examined on Leica DM IRB or Zeiss Axiovert $200 \mathrm{M}$ microscopes using Axiovision software. For quantification, at least 100 nuclei were counted per sample and analyzed using ImageJ software.

\subsection{Viability assay}

Cells were grown to $80 \%$ confluency, trypsinized, pelleted at $300 \times \mathrm{g}$ for $5 \mathrm{~min}$ at room temperature (RT) and resuspended in fresh medium to a concentration of $1 \times 10^{6} \mathrm{cells} / \mathrm{ml}$. Cell suspensions of $1 \mathrm{ml}$ each were transferred to $1.5 \mathrm{ml}$ reaction tubes and pelleted at $300 \times g$ for $5 \mathrm{~min}$ at RT. Cells were resuspended in $1 \mathrm{ml}$ of PBS containing CEES final concentrations as indicated. The treatment with CEES was performed in a heating block at $37^{\circ} \mathrm{C}$ for $1 \mathrm{~h}$. Cells were pelleted at $300 \times \mathrm{g}$ for $5 \mathrm{~min}$ at RT, washed with PBS and resuspended in cell culture medium. Samples were seeded into 96 well plates in quadrupli cates ( 6000 cells/well) and incubated under standard cell culture conditions for $72 \mathrm{~h}$. After discarding the medium, $100 \mu \mathrm{l}$ of culture medium containing 10\% Alamar Blue reagent (Invitrogen) was added to each well and after $23 \mathrm{~h}$ of incubation $\left(37^{\circ} \mathrm{C}, 5 \% \mathrm{CO}_{2}\right)$ the fluorescence signal was measured at $550 \mathrm{~nm}$ excitation wave length and $590 \mathrm{~nm}$ emission wavelength. Results were expressed as relative fluorescence of the sample to the fluorescence of control [in \%].

\subsection{Fluorimetric alkaline DNA unwinding assay (FADU assay)}

DNA strand breaks were assessed by the automated FADU assay as described previously (Debiak et al., 2011). Briefly, HaCaT cells were treated with different doses of CEES according to the protocol \#3, washed with ice cold PBS and resuspended in suspension buffer (10 mM Na phosphate buffer, pH 7.4; $0.25 \mathrm{M}$ meso inositol; $1 \mathrm{mM} \mathrm{MgCl} 2$ ) at the final concentration of $2 \times 10^{5}$ cells $/ \mathrm{ml}$. $70 \mu \mathrm{l}$ of the cell suspension was transferred on ice into 96 well plates and lysed with $70 \mu$ l lysis buffer ( $9 \mathrm{M}$ urea, $10 \mathrm{mM} \mathrm{NaOH}, 2.5 \mathrm{mM} \mathrm{1,2}$ cyclohexanedinitrilotetraacetic acid, $0.1 \%$ SDS). Samples were incubated for $12 \mathrm{~min}$ at $0^{\circ} \mathrm{C}$. Pre chilled alkaline buffer (42.5\% lysis buffer, $0.2 \mathrm{M} \mathrm{NaOH}$ ) was overlaid on top of the cell lysate. Alkaline unwinding was performed at $30^{\circ} \mathrm{C}$ for $60 \mathrm{~min}$. Unwinding was stopped with $140 \mu$ l neutralization buffer (1 M glucose, $14 \mathrm{mM}$ $\beta$ mercaptoethanol). Following incubation at $22^{\circ} \mathrm{C}$ for $30 \mathrm{~min}$, $156 \mu$ l SybrGreen solution (1:8333 v/v in $\mathrm{H}_{2} 0$; MoBiTec, Göttingen,
Germany) was added. Samples were mixed by automatic pipetting up and down. Fluorescence was measured in a 96 well plate reader by excitation at $492 \mathrm{~nm}$ and emission at $520 \mathrm{~nm}$. For each sample an internal control of the DNA content was incorporated without alkaline unwinding process. Here the unwinding was prevented by adding of neutralization buffer to samples prior to the addition of alkaline buffer.

The amount of double stranded DNA was calculated as (fluorescence of sample/fluorescence of sample without unwinding) $\times 100 \%$.

\subsection{PARP1 knock down}

RNA interference experiments were performed by transfecting cells with ON Targetplus siRNA targeting PARP1 or control siRNA (ThermoScientific). After $48 \mathrm{~h}$, cells were harvested for Western blot analysis or treated with CEES for PAR analysis by immunoflu orescence microscopy.

\subsection{SDS PAGE and Western blotting}

Cells $\left(1 \times 10^{6}\right.$ cells $\left./ \mathrm{ml}\right)$ were resuspended in hot $1 \times$ Roti Load 1 buffer (Roth) and sonicated twice for $30 \mathrm{~s}$ with $50 \%$ pulse for 30 cycles. Proteins were separated by SDS PAGE and were transferred on ECL nitrocellulose membrane (GE Healthcare). Membranes were blocked in $5 \%$ milk powder in TBS $(15 \mathrm{mM} \mathrm{NaCl}, 1 \mathrm{mM}$ Tris Base, $\mathrm{pH}$ adjusted to 8.0 ) for $1 \mathrm{~h}$ at $\mathrm{RT}$ and incubated with anti PARP1 antibody (CII 10; $1: 300,4^{\circ} \mathrm{C}$, overnight). Then, membranes were washed and incubated with secondary antibody goat anti mouse HRP coupled (1:2000; DakoCytomation) for $1 \mathrm{~h}$ at RT. Detection was performed by means of ECL Advance/Prime Western Blotting Detection Kit (GE Healthcare) according to the manu facturer's protocol. Images were acquired with ImageQuant LAS 4000 mini (GE Healthcare).

\subsection{Statistical analysis}

Statistical analysis of data was performed with GraphPad Prism (GraphPad Software) software. The results were analyzed using Student's $t$ test or 2 way ANOVA as appropriate.

\section{Results and discussion}

\subsection{Optimization of the CEES treatment protocol for cellular detection of poly(ADP ribose)}

Since CEES undergoes rapid hydrolysis in aqueous solutions, various treatment protocols for CEES have been reported using non aqueous solvents such as DMSO, ethanol, or acetone [e.g., (Jain et al., 2011; Paromov et al., 2011a; Tewari Singh et al., 2010, 2014)]. Furthermore, conditions reported in the literature vary by treatment temperature, time, pre dilution factors and medium in which the treatment is performed. In order to define optimal CEES treatment conditions for the detection of PAR formation in HaCaT cells we set out to test different experimental protocols. Therefore, we treated cells for 10 min with CEES pre diluted in different stock solutions and analyzed PAR formation via immuno fluorescence microscopy based on a previously established protocol (Küpper et al., 1996). As it is evident from Fig. 1, applying CEES in DMSO in a $100 \times$ concentrated stock solution (protocol \#1) did result in PAR formation only in a minor subfraction of cells, potentially due to DMSO hygroscopicity and related CEES hydrolysis. Based on available literature information, we 
rationalized that CEES diluted in ethanol supplemented with $0.5 \%$ $\mathrm{HCl}$ may serve as a better solvent, since the presence of $\mathrm{HCl}$ is expected to stabilize CEES by shifting the hydrolysis equilibrium from 2 hydroxyethyl ethyl sulfide towards CEES (Wang et al., 2012). In a first approach, we prepared CEES as a $1000 \times$ stock solution, which was then added directly to the cell culture medium (protocol \#2). As it is evident from Fig. 1, considerably more cells displayed detectable levels of PAR formation, however responding cells were unevenly distributed throughout the culture dish. While cells in some regions of the culture dish showed a strong staining for PAR, the signal intensity in other regions did not differ significantly from control cells. In contrast, PARP activation in cells treated with hydrogen peroxide $\left(\mathrm{H}_{2} \mathrm{O}_{2}\right)$ was regularly distributed. Interestingly, CEES treated cells showing high PAR levels were localized in defined groups/regions. Since a detailed analysis of microscopic slides excluded any clonal effects of cells growth, which might have accounted for this phenomenon, we concluded that these results were presumably caused by an unequal distribution of CEES in the culture dishes (Fig. 1). To the best of our knowledge, such difficulties in treating cells with CEES have not been reported before. First the unique combination of different factors such as very short assessment time and a very sensitive endpoint analyzed at a single cell level used in our study (i.e., PAR formation analyzed by immunofluorescence microscopy) resulted in the appearance of this complication, which potentially also leads to research artefacts with regards to other cellular end points.

In the following the treatment protocol was optimized to better take into account the physico chemical properties of CEES. Therefore, CEES was pre diluted in $\mathrm{EtOH} / \mathrm{HCl}$ in a $100 \times$ stock solution. Furthermore, a CEES working solution was prepared by adding the CEES stock solution to pre warmed PBS $\left(37^{\circ} \mathrm{C}\right)$ shortly before cells were exposed. After short but intensive mixing, the CEES solution was immediately added to the adherent cells (protocol \#3). As it is evident from Fig. 1, using this protocol, PAR formation occurred in all cells exposed to CEES. Furthermore, signal intensities were considerable higher as in cells treated with CEES diluted in DMSO, thereby significantly improving the sensitivity of the method. Importantly, solvent controls did not show any PAR formation above background levels (Fig. 1).

\subsection{Impact of treatment protocol on CEES toxicity}

Following the observation that individual CEES treatment protocols significantly affect the intensity and homogeneity of cellular PAR formation, individual treatment protocols were analyzed with regards to overall cytotoxicity. Previous studies of CEES induced cytotoxicity in HaCaT cells reported LC50 values differing by at least one order of magnitude within a range of 0.25 $2 \mathrm{mM}$ depending on treatment time and conditions applied (Paromov et al., 2011a,b; Tewari Singh et al., 2010, 2014). One may assume that cell cultures displaying heterogeneous areas of PAR formation throughout the culture dish (Fig. 1), were unequally exposed to CEES, which may lead to an overall lower toxicity compared to cultures showing uniform PAR formation, which indicates homogenous exposure of CEES to all cells within a culture dish. The three different CEES treatment protocols as mentioned above (protocols \#1 3) were analyzed for their ability to induce cytotoxicity in HaCaT cell cultures. To this end, cells were treated with different doses of CEES for $60 \mathrm{~min}$ and cell viability was measured $72 \mathrm{~h}$ after treatment (Fig. 2). As supposed, solvent conditions had a substantial impact on CEES induced cytotoxicity. The LC50 of the $1000 \times$ concentrated $\mathrm{EtOH} / \mathrm{HCl}$ CEES stock solution (protocol \#2) was in the range of $1 \mathrm{mM}$ and therefore almost four times as high as compared to the $100 \times$ concentrated stock solutions with CEES dissolved in $\mathrm{EtOH} / \mathrm{HCl}$ or DMSO (LC50 $~ 0.25$ $\mathrm{mM}$, protocols \#1 and \#3). It can be assumed that in cultures

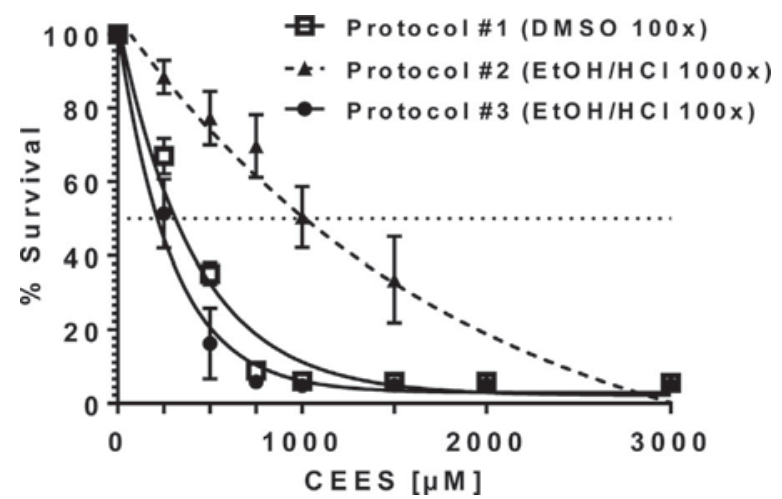

Fig. 2. Treatment protocol affects CEES-induced cytotoxicity in HaCaT cells. $\mathrm{HaCaT}$ cells were treated in suspension with different concentrations of CEES using different treatment protocols as indicated and described in the text body. Cells were incubated with CEES for $60 \mathrm{~min}$, washed with PBS, and cell viability was analyzed $72 \mathrm{~h}$ after treatment using an Alamar Blue assay. $n=3 \pm$ SD. A non-linear curve fit model was applied.

treated by protocol \#2, individual cells were able to survive the treatment with doses as high as $3 \mathrm{mM}$ of CEES, whereas the lower pre dilution (i.e., protocols \#1 and \#3) resulted in almost $100 \%$ toxicity at a concentration of $1 \mathrm{mM}$. In contrast to the dilution factor, the choice of the solvent between DMSO (protocol \#1) and $\mathrm{EtOH} / \mathrm{HCl}$ (protocol \#3) did not significantly influence cytotoxicity, indicating that in both solvents CEES has been distributed uniformly throughout the culture dish. However, the differential results with regards to the PARylation response (c.f. Fig. 1) suggest that depending on the solvent used, CEES exhibits significantly different toxicokinetics in the short term, which could significant ly influence measurements of molecular and cellular end points of toxicity.

\subsection{Application of the optimized CEES treatment prototocol for the} analysis of PARP activation in HaCaT cells

After defining optimal treatment conditions, we characterized the CEES induced PARylation response in $\mathrm{HaCaT}$ in detail. Several previous studies reported PAR formation after SM treatment, however at present those remain incidental and lack any systematic time and dose dependent analysis (Debiak et al., 2009; Hinshaw et al., 1999; Kehe et al., 2008; Rosenthal et al., 1998). In terms of CEES induced PAR formation, Paromov analyzed PARP activity in HaCaT cell extracts using biotinylated $\mathrm{NAD}^{+}$, leading to a maximum of a $\sim 10$ fold in signal intensities at $5 \mathrm{mM}$ CEES treatment (Paromov et al., 2011a). However, to the best of our knowledge, intracellular PAR formation in intact cells with endogenous $\mathrm{NAD}^{+}$as substrate has so far not been reported upon CEES treatment. Since cellular PARylation is a very dynamic process, due to several PAR degrading enzymes such as poly(ADP ribose) glycohydrolase (PARG), we performed a time course analysis of PAR formation in intact HaCaT cells upon CEES treatment. As it is evident from Fig. 3A, PAR activation was detected as soon as $5 \mathrm{~min}$ after CEES treatment. PAR levels peaked at 10 min post treatment and almost complete PAR degradation was observed $30 \mathrm{~min}$ after treatment. Next we focused on the time point of maximum PAR formation (i.e., $10 \mathrm{~min}$ post treatment) to analyze CEES PARylation dose response relation ships. We demonstrated a dose dependent increase in cellular PAR formation (Fig. 3B). The increase in cellular PAR was observed at $1 \mathrm{mM}$ CEES and $3 \mathrm{mM}$ CEES resulted in circa eight fold increase of PAR concentration in comparison to the untreated control. However, although a highly toxic dose was applied the PARP activation was not as high as the activation upon hydrogen peroxide treatment. 
A

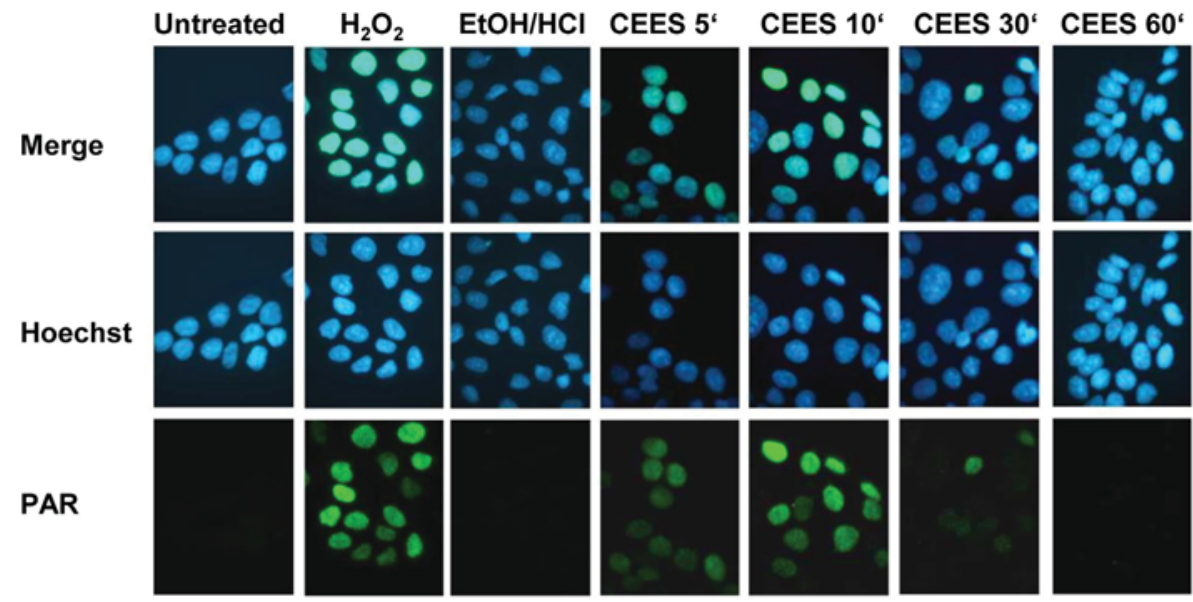

B

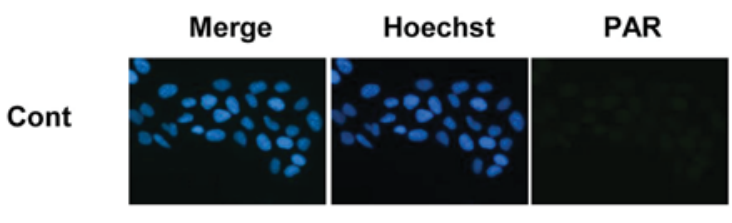

$\mathrm{H}_{2} \mathrm{O}_{2}$

(0.1 mM)
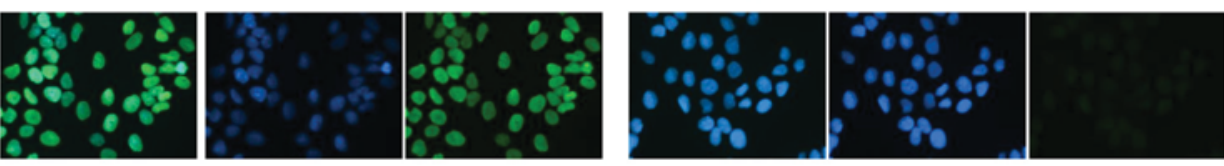

CEES (0.5 mM)
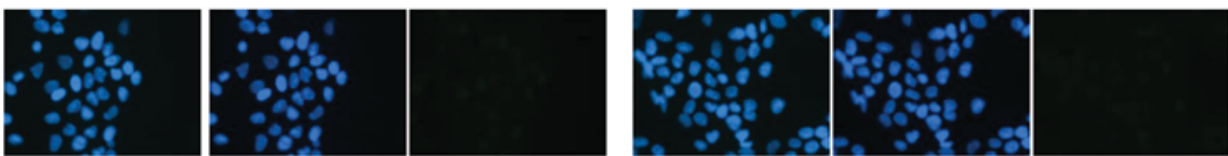

CEES

(2 mM)
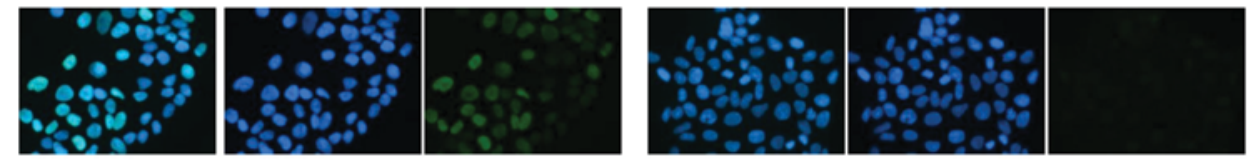

CEES

(5 mM)
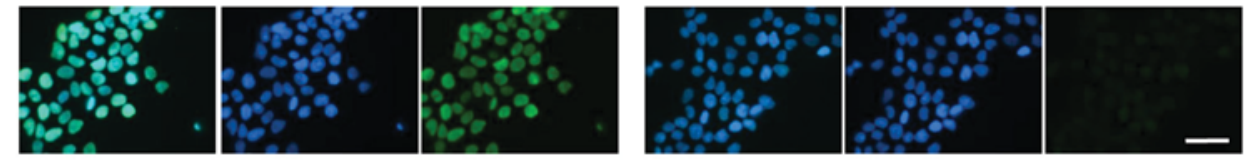

C

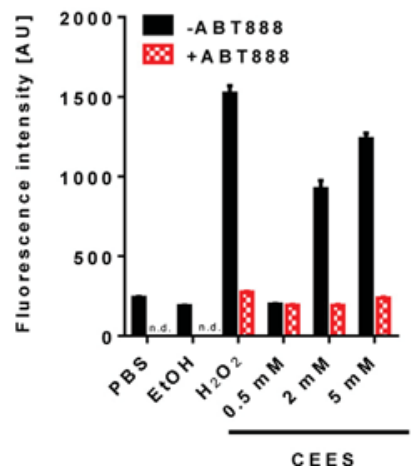

D

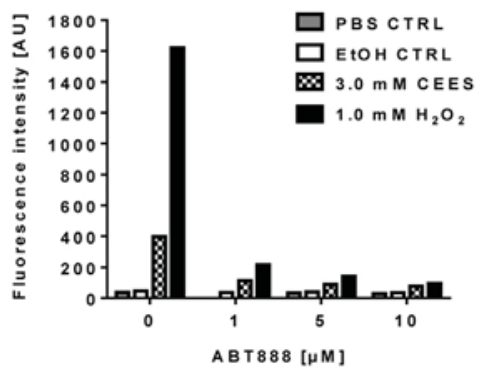

$24 \mathrm{~h}$ preincubation

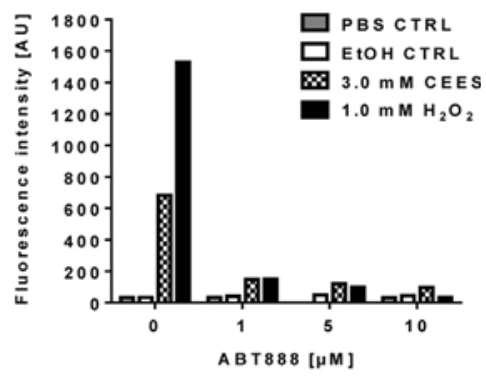

Fig. 3. Time and dose-dependent analysis of cellular PARylation response upon CEES treatment.

(A) Time-dependent cellular PARylation in HaCaT cells after treatment with CEES as analyzed by epi-immunofluorescence microscopy. Cells were fixed at time points as indicated, and stained for cellular PAR using the anti-PAR-specific $\mathrm{mAB}(10 \mathrm{H}) \cdot \mathrm{H}_{2} \mathrm{O}_{2}(1 \mathrm{mM})$ treatment for 5 min served as a positive control. (B) Dose response relationship of CEES treatment and PAR formation and its inhibition by the pharmacological PARP inhibitor ABT888. Cells were treated with $\mathrm{CEES}$ (for 10 min) or $\mathrm{H}_{2} \mathrm{O}_{2}$ (for 5 min) at concentrations as indicated. Scale bar indicates $50 \mu \mathrm{m}$. (C) Densitometric analysis of immunofluorescence data shown in B. PBS refers to PBS control, EtOH to EtOH/HCl solvent control ( $1 \%(v / v)$ final concentration. (D) Determination of concentrations and pre-incubation times of $A B T 888$ treatment for effective inhibition of $\mathrm{CEES}$ - and $\mathrm{H}_{2} \mathrm{O}_{2}-$ induced PARylation.

In order to prove the specificity of the $10 \mathrm{H}$ derived PAR signal in fluorescence microscopy, we suppressed PAR formation in HaCaT cells by using the clinically relevant pharmacological PARP inhibitor ABT888 (also known as veliparib). These experiments revealed that PAR formation was almost completely inhibited after genotoxic treatment with CEES or $\mathrm{H}_{2} \mathrm{O}_{2}$, when $\mathrm{HaCaT}$ cells were 


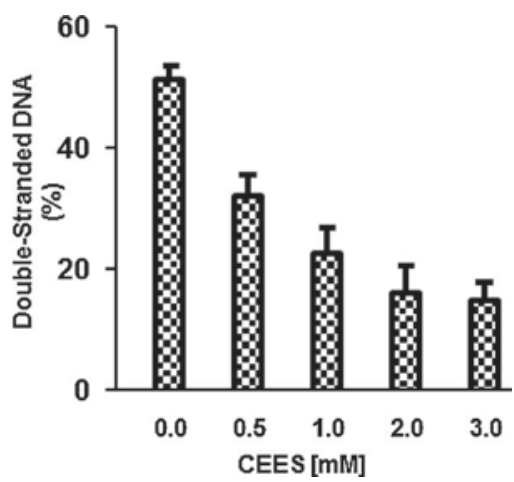

Fig. 4. HaCaT cells exposed to CEES incorporate DNA strand breaks in dose dependent manner.

$\mathrm{HaCaT}$ cells were treated with CEES for $60 \mathrm{~min}$, washed with PBS and preceded immediately by the automated FADU assay. The degree of DNA unwinding is related with the amount of DNA strand breaks. Data represents mean values $\pm S D$ of three independent experiments $(n=3)$.

pre incubated for 30 min with $1 \mu \mathrm{M}$ of ABT888 (Fig 3B D). In this respect, it is important to note that PARP inhibitors are discussed as a potential treatment option to reduce mustard related patholo gies in exposed victims (Debiak et al., 2009). However, the moderate PARP activation induced by CEES presumably leads only to minor changes in intracellular $\mathrm{NAD}^{+}$concentrations, which speaks against the hypothesis that SM causes massive PARP activation resulting in ATP depletion, necrotic cell death, and subsequent tissue inflammation. For a detailed cell culture study characterizing the PARylation response induced by bifunctional mustards as well as a discussion of the therapeutic potential of PARP inhibitors in SM related pathologies the reader is referred to an accompanying paper (Mangerich et al., 2016)

It is interesting to note that PAR formation after $\mathrm{H}_{2} \mathrm{O}_{2}$ peaks already at 5 min post treatment (Martello et al., 2013), whereas maximum PARylation was observed 10 min post CEES treatment. One possibility for the different PARylation dynamics are substance specific toxicokinetics. Alternatively, different PARy lation dynamics may be a direct consequences of the different types of DNA lesions formed, i.e., $\mathrm{H}_{2} \mathrm{O}_{2}$ induces mainly oxidative damage and strand breaks, while CEES induces mainly DNA alkylation, without being thought to induce DNA strand breaks directly. Previous reports showed that CEES induced DNA mono adducts are predominantly repaired by BER and NER pathways (Jowsey et al., 2009; Matijasevic et al., 2001). PARylation plays an active role in both repair pathway, potentially by being activated through DNA strand breaks introduced by endonucleases as intermediates during the repair processes (Fischer et al., 2014; Pines et al., 2013, 2012; Robert et al., 2013). Thus, even though mustards do not induce DNA strand breaks directly, strand breaks can occur in response to mustard treatment by enzymatic processes that actively introduce them in the course of repair processes, thereby triggering PARP activation. Potential CEES induced DNA strand break formation in HaCaT cells was assessed by the automated FADU assay (Fig. 4). The assay makes use of the direct relationship between the extent of DNA unwinding under alkaline conditions and the number of DNA strand breaks. The acquisition is based on detection of remaining double stranded DNA by means of a fluorescent DNA intercalating dye. Thus the relative amount of double stranded DNA is inversely related with the number of DNA strand breaks. HaCaT cells were treated for 60 min with different doses of CEES and analyzed immediately thereafter. During the processing, cells were kept on ice to suppress DNA repair. CEES treatment led to a dose dependent DNA strand break induction, which was evident already the lowest applied dose of $0.5 \mathrm{mM}$ CEES. DNA strand break formation by CEES was analyzed previously also in human PBMCs (Debiak et al., 2011). In non proliferating cells the unwinding takes place only at chromosome ends. Thus the amount of double stranded DNA in control PBMC is about $90 \%$, whereas in proliferating $\mathrm{HaCaT}$ cells, where replication dependent breaks act as additional unwinding initiators, only 50 60\% DNA remains double stranded. The low background in control PBMCs influenced substantially the assay sensitivity and DNA breaks in PBMC were detected already after treatment with $50 \mu \mathrm{M}$ CEES. A dose dependent increase in DNA damage observed after CEES treatment in PBMC suggests a replication independent mechanism of DNA break induction such as direct reaction of CEES, ROS, BER or NER activity (Debiak et al., 2011).

In addition to PARP1, PARP2 and PARP3 can also be activated upon certain genotoxic stimuli. While PARP1 is involved in most DNA repair processes, such as base and nucleotide excision repair, as well as DNA strand break repair, PARP2 appears to contribute mainly to BER and single strand break repair and PARP3 to double strand break repair (Beck et al., 2014; De Vos et al., 2012). To test the hypothesis that the PARP1 homologue is responsible for PAR formation upon CEES treatment, presumably through its role in BER and NER, we silenced PARP1 expression by transfecting HaCaT cells with siRNA targeting PARP1 transcripts. The efficiency of the PARP1 knockdown was over $80 \%$ (Fig. 5A). Of note, PAR formation after CEES treatment correlated directly with the PARP1 protein level, indicating that of the three known DNA damage dependent
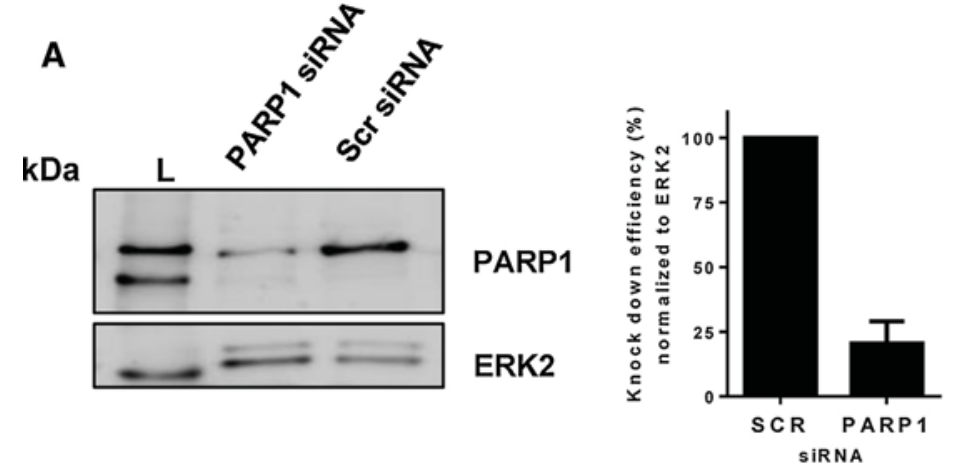

B

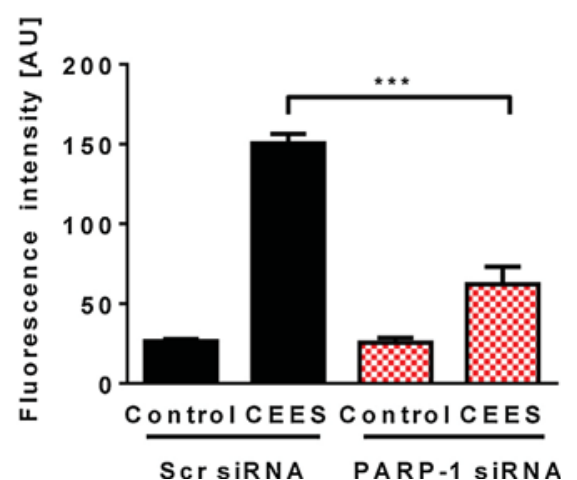

Fig. 5. PARP1 is responsible for PAR synthesis after CEES treatment

(A) and (B) PARP1 is the main contributor to CEES-induced PARylation in HaCaT cells. Cells were transfected with siRNA targeting PARP1 (PARP1 siRNA) or control siRNA (scr siRNA). (A) Left. Western Blot analysis of PARP1 protein levels $48 \mathrm{~h}$ upon siRNA transfection. Detection of ERK2 served as loading control. Right. Densitometric analysis of 3 independent experiments. (B) Analysis of PARylation response $48 \mathrm{~h}$ after siRNA transfection and treatment of cells with $3 \mathrm{mM} C E E S$ for 10 min. Data represent means \pm SD from 3 independent experiments. Statistical analysis was performed using a Student's $t$ test. ${ }^{* * *} P<0.001$. 
PARPs (i.e., PARP1 3), PARP1 activation is responsible for the bulk of PAR production upon CEES treatment.

\section{Conclusions}

In conclusion, this study provides a detailed analysis of the cellular PARylation response in HaCaT cells upon treatment with the sulfur mustard derivative CEES. By comparing different treatment protocols we could optimize experimental conditions, which allow the analysis of a homogenous, time and dose dependent PARylation response on a single cell level using immuno chemical based epifluorescence microscopy. Using siRNA directed against PARP1, we provide evidence that PARP1 is the main contributor to CEES induced PARylation. Furthermore, we define treatment conditions to effectively inhibit CEES induced PARylation by using the clinically relevant PARP inhibitor ABT888 (veliparib). Taken together, this study forms an experimental basis to study the molecular mechanisms of mustard induced PARP1 activation and its cellular consequences. It will be interesting to apply the optimized methodology as established in this work to study the PARylation response in cells treated with bifunctional sulfur and nitrogen mustards. This will have significant implica tions towards the question if PARP inhibitors may be used as a therapeutic option to mitigate SM induced pathologies, as suggested by several animal studies (Cowan et al., 2003; Gross et al., 1985; Mol et al., 1991; Yourick et al., 1991, 1993; Zhang et al., 1995) or to sensitize cancer cells to nitrogen mustard treatment, as tested in preclinical and clinical settings (Donawho et al., 2007; Norris et al., 2014). Several of these aspects are addressed in in an accompanying article (c.f. Mangerich et al., 2016).

\section{Acknowledgements}

We thank Matthias Birtel for his contributions to experiments included in Fig. 3. This work was supported by the German Federal Ministry of Defense (Grant E/UR3G/AG001/94804).

\section{References}

Beck, C., Boehler, C., Guirouilh Barbat, J., Bonnet, M.E., Illuzzi, G., Ronde, P., Gauthier, L.R., Magroun, N., Rajendran, A., Lopez, B.S., Scully, R., Boussin, F.D., Schreiber, V., Dantzer, F., 2014. PARP3 affects the relative contribution of homologous recombination and nonhomologous end-joining pathways. Nucleic Acids Res. 42 (9), 5616-5632.

Bennett, R.A., Behrens, E., Zinn, A., Duncheon, C., Lamkin, T.J., 2014. Mustard gas surrogate, 2-chloroethyl ethylsulfide (2-CEES), induces centrosome amplification and aneuploidy in human and mouse cells: 2-CEES induces centrosome amplification and chromosome instability. Cell Biol. Toxicol. 30, 195-205.

Boukamp, P., Petrussevska, R.T., Breitkreutz, D., Hornung J., Markham, A., Fusenig, N. E., 1988. Normal keratinization in a spontaneously immortalized aneuploid human keratinocyte cell line. J. Cell Biol. 106, 761-771.

Cowan, F.M., Broomfield, C.A., Lenz, D.E., Smith, W.J., 2003. Putative role of proteolysis and inflammatory response in the toxicity of nerve and blister chemical warfare agents: implications for multi-threat medical countermeasures. J. Appl. Toxicol. 23, 177-186.

De Vos, M., Schreiber, V., Dantzer, F., 2012. The diverse roles and clinical relevance of PARPs in DNA damage repair: current state of the art. Biochem. Pharmacol. 84, 137-146.

Debiak, M., Kehe, K., Bürkle, A., 2009. Role of poly(ADP-ribose) polymerase in sulfur mustard toxicity. Toxicology 263, 20-25.

Debiak, M., Panas, A., Steinritz, D., Kehe, K., Bürkle, A., 2011. High-throughput analysis of DNA interstrand crosslinks in human peripheral blood mononuclear cells by automated reverse FADU assay. Toxicology 280, 53-60.

Donawho, C.K., Luo, Y., Luo, Y., Penning, T.D., Bauch, J.L., Bouska, J.J., Bontcheva-Diaz, V.D., Cox, B.F., DeWeese, T.L., Dillehay, L.E., Ferguson, D.C., Ghoreishi-Haack, N.S., Grimm, D.R., Guan, R., Han, E.K., Holley-Shanks, R.R., Hristov, B., Idler, K.B., Jarvis, K., Johnson, E.F., Kleinberg, L.R., Klinghofer, V., Lasko, L.M., Liu, X., Marsh, K.C., McGonigal, T.P., Meulbroek, J.A., Olson, A.M., Palma, J.P., Rodriguez, L.E., Shi, Y., Stavropoulos, J.A., Tsurutani, A.C., Zhu, G.D., Rosenberg, S.H., Giranda, V.L., Frost, D.J., 2007. ABT-888, an orally active poly(ADP-ribose) polymerase inhibitor that potentiates DNA-damaging agents in preclinical tumor models. Clin. Cancer Res. 13, 2728-2737.
Fischer, J.M., Popp, O., Gebhard, D., Veith, S., Fischbach, A., Beneke, S., Leitenstorfer, A., Bergemann, J., Scheffner, M., Ferrando-May, E., Mangerich, A., Bürkle, A., 2014. Poly(ADP-ribose)-mediated interplay of XPA and PARP1 leads to reciprocal regulation of protein function. FEBS J. 281, 3625-3641.

Gross, C.L., Meier, H.L., Papirmeister, B., Brinkley, F.B., Johnson, J.B., 1985. Sulfur mustard lowers nicotinamide adenine dinucleotide concentrations in human skin grafted to athymic nude mice. Toxicol. Appl. Pharmacol. 81, 85-90.

Hinshaw, D.B., Lodhi, I.J., Hurley, L.L., Atkins, K.B., Dabrowska, M.I., 1999. Activation of poly [ADP-Ribose] polymerase in endothelial cells and keratinocytes: role in an in vitro model of sulfur mustard-mediated vesication. Toxicol. Appl. Pharmacol. 156, 17-29.

Inturi, S., Tewari-Singh, N., Gu, M., Shrotriya, S., Gomez, J., Agarwal, C., White, C.W., Agarwal, R., 2011. Mechanisms of sulfur mustard analog 2-chloroethyl ethyl sulfide-induced DNA damage in skin epidermal cells and fibroblasts. Free Radic. Biol. Med. 51, 2272-2280.

Jain, A.K., Tewari-Singh, N., Orlicky, D.J., White, C.W., Agarwal, R., 2011. 2Chloroethyl ethyl sulfide causes microvesication and inflammation-related histopathological changes in male hairless mouse skin. Toxicology 282, 129-138.

Jowsey, P.A., Williams, F.M., Blain, P.G., 2009. DNA damage, signalling and repair after exposure of cells to the sulphur mustard analogue 2-chloroethyl ethyl sulphide. Toxicology 257, 105-112.

Kawamitsu, H., Hoshino, H., Okada, H., Miwa, M., Momoi, H., Sugimura, T., 1984. Monoclonal antibodies to poly(adenosine diphosphate ribose) recognize different structures. Biochemistry 23, 3771-3777.

Kehe, K., Szinicz, L., 2005. Medical aspects of sulphur mustard poisoning. Toxicology 214, 198-209.

Kehe, K., Raithel, K., Kreppel, H., Jochum, M., Worek, F., Thiermann, H., 2008. Inhibition of poly(ADP-ribose) polymerase (PARP) influences the mode of sulfur mustard (SM)-induced cell death in HaCaT cells. Arch. Toxicol. 82, 461470.

Kehe, K., Balszuweit, F., Steinritz, D., Thiermann, H., 2009. Molecular toxicology of sulfur mustard-induced cutaneous inflammation and blistering. Toxicology 263, 12-19.

Kehe, K., Schrettl, V., Thiermann, H., Steinritz, D., 2013. Modified immunoslotblot assay to detect hemi and sulfur mustard DNA adducts. Chem. Biol. Interact..

Küpper, J.H., Muller, M., Bürkle, A., 1996. Trans-dominant inhibition of poly(ADPribosyl)ation potentiates carcinogen induced gene amplification in SV40transformed Chinese hamster cells. Cancer Res. 56 (12), 2715-2717.

Ludlum, D.B., Papirmeister, B., 1986. DNA modification by sulfur mustards and nitrosoureas and repair of these lesions. Basic Life Sci. 38, 119-125.

Ludlum, D.B., Austin-Ritchie, P., Hagopian, M., Niu, T.Q., Yu, D., 1994. Detection of sulfur mustard-induced DNA modifications. Chem. Biol. Interact. 91, 39-49.

Mangerich, A., Bürkle, A., 2012. Pleiotropic cellular functions of PARP1 in longevity and aging: genome maintenance meets inflammation. Oxid. Med. Cell. Longev. 321653.

Mangerich, A., Esser, C., 2014. Chemical warfare in the First World War: reflections 100 years later. Arch. Toxicol. 88, 1909-1911.

Mangerich, A., Debiak, M., Birtel, M., Ponath, V., Balszuweit, F., Lex, K., Martello, R., Burckhardt-Boer, W., Strobelt, R., Siegert, M., Thiermann, H., Steinritz, D., Schmidt, A., Bürkle, A., 2016. Sulfur and nitrogen mustards induce characteristic poly(ADP-ribosyl)ation responses in $\mathrm{HaCaT}$ keratinocytes with distinctive cellular consequences. Toxicol. Lett. 244, 56-71.

Martello, R., Mangerich, A., Sass, S., Dedon, P.C., Bürkle, A., 2013. Quantification of cellular poly(ADP-ribosyl)ation by stable isotope dilution mass spectrometry reveals tissue- and drug-dependent stress response dynamics. ACS Chem. Biol. 8 (7), 1567-1575.

Matijasevic, Z., Precopio, M.L., Snyder, J.E., Ludlum, D.B., 2001. Repair of sulfur mustard-induced DNA damage in mammalian cells measured by a host cell reactivation assay. Carcinogenesis 22, 661-664.

Meier, H.L., Gross, C.L., Papirmeister, B., 1987. 2,2'-Dichlorodiethyl sulfide (sulfur mustard) decreases NAD+ levels in human leukocytes. Toxicol. Lett. 39, 109-122.

Mol, M.E., de Vries, R., Kluivers, A.W., 1991. Effects of nicotinamide on biochemical changes and microblistering induced by sulfur mustard in human skin organ cultures. Toxicol. Appl. Pharmacol. 107, 439-449.

Norris, R.E., Adamson, P.C., Nguyen, V.T., Fox, E., 2014. Preclinical evaluation of the PARP inhibitor, olaparib, in combination with cytotoxic chemotherapy in pediatric solid tumors. Pediatr. Blood Cancer 61, 145-150.

Papirmeister, B., Gross, C.L., Meier, H.L., Petrali, J.P., Johnson, J.B., 1985. Molecular basis for mustard-induced vesication. Fundam. Appl. Toxicol. 5 S134-S149.

Paromov, V., Brannon, M., Kumari, S., Samala, M., Qui, M., Smith, M., Stone, W.L., 2011a. Sodium pyruvate modulates cell death pathways in HaCaT keratinocytes exposed to half-mustard gas. Int. J. Toxicol. 30, 197-206.

Paromov, V., Kumari, S., Brannon, M., Kanaparthy, N.S., Yang, H., Smith, M.G., Stone, W.L., 2011b. Protective effect of liposome-encapsulated glutathione in a human epidermal model exposed to a mustard gas analog. J. Toxicol. 2011 doi:http://dx. doi.org/10.1155/2011/109516 109516 .

Pines, A., Vrouwe, M.G., Marteijn, J.A., Typas, D., Luijsterburg, M.S., Cansoy, M., Hensbergen, P., Deelder, A., de Groot, A., Matsumoto, S., Sugasawa, K., Thoma, N., Vermeulen, W., Vrieling, H., Mullenders, L., 2012. PARP1 promotes nucleotide excision repair through DDB2 stabilization and recruitment of ALC1. J. Cell Biol. 199, 235-249. 
Pines, A Mullenders, L.H. van Attikum, H., Luijsterburg, M.S., 2013. Touching base with PARPs: moonlighting in the repair of UV lesions and double-strand breaks. Trends Biochem. Sci. 38, 321-330.

Robert, I., Karicheva, O., Reina San Martin, B., Schreiber, V., Dantzer, F., 2013. Functional aspects of PARylation in induced and programmed DNA repair processes: preserving genome integrity and modulating physiological events. Mol. Aspects Med. 34, 1138-1152.

Rosenthal, D.S., Simbulan-Rosenthal, C.M., Iyer, S., Spoonde, A., Smith, W., Ray, R., Smulson, M.E., 1998. Sulfur mustard induces markers of terminal differentiation and apoptosis in keratinocytes via a Ca2+-calmodulin and caspase-dependent pathway. J. Invest. Dermatol. 111, 64-71.

Rouleau, M., Patel, A., Hendzel, M.J., Kaufmann, S.H., Poirier, G.G., 2010. PARP inhibition: PARP1 and beyond. Nat. Rev. Cancer 10, 293-301.

Tewari-Singh, N., Rana, S., Gu, M. Pal, A., Orlicky, D.J., White, C.W., Agarwal, R., 2009. Inflammatory biomarkers of sulfur mustard analog 2-chloroethyl ethyl sulfideinduced skin injury in SKH-1 hairless mice. Toxicol. Sci. 108, 194-206.

Tewari-Singh, N., Gu, M., Agarwal, C., White, C.W., Agarwal, R., 2010. Biological and molecular mechanisms of sulfur mustard analogue-induced toxicity in JB6 and HaCaT cells: possible role of ataxia telangiectasia-mutated/ataxia
telangiectasia-Rad3-related cell cycle checkpoint pathway. Chem. Res. Toxicol. 23, 1034-1044

Tewari-Singh, N., Inturi, S., Jain, A.K., Agarwal, C., Orlicky, D.J., White, C.W., Agarwa R., Day, B.J., 2014. Catalytic antioxidant AEOL 10150 treatment ameliorates sulfur mustard analog 2-chloroethyl ethyl sulfide-associated cutaneous toxic effects. Free Radic. Biol. Med. 72, 285-295.

Wang, Q.Q., Begum, R.A., Day, V.W., Bowman-James, K., 2012. Sulfur, oxygen, and nitrogen mustards: stability and reactivity. Org. Biomol. Chem. 10, 8786-8793.

Yourick, J.J., Clark, C.R., Mitcheltree, L.W., 1991. Niacinamide pretreatment reduces microvesicle formation in hairless guinea pigs cutaneously exposed to sulfur mustard. Fundam. Appl. Toxicol. 17, 533-542.

Yourick, J.J., Dawson, J.S., Benton, C.D., Craig, M.E., Mitcheltree, L.W., 1993. Pathogenesis of 2,2'-dichlorodiethyl sulfide in hairless guinea pigs. Toxicology 84, 185-197.

Zhang, Z., Riviere, J.E., Monteiro-Riviere, N.A., 1995. Evaluation of protective effects of sodium thiosulfate, cysteine, niacinamide and indomethacin on sulfur mustard-treated isolated perfused porcine skin. Chem. Biol. Interact. 96, 249-262. 